\title{
A consideration of publication-derived immune-related associations in Coronavirus and related lung damaging diseases
}

Nophar Geifman ${ }^{1 *}$ (1) and Anthony D. Whetton ${ }^{2,3}$

\begin{abstract}
Background: The severe acute respiratory syndrome virus SARS-CoV-2, a close relative of the SARS-CoV virus, is the cause of the recent COVID-19 pandemic affecting, to date, over 14 million individuals across the globe and demonstrating relatively high rates of infection and mortality. A third virus, the $\mathrm{H} 5 \mathrm{~N} 1$, responsible for avian influenza, has caused infection with some clinical similarities to those in COVID-19 infections. Cytokines, small proteins that modulate immune responses, have been directly implicated in some of the severe responses seen in COVID-19 patients, e.g. cytokine storms. Understanding the immune processes related to COVID-19, and other similar infections, could help identify diagnostic markers and therapeutic targets.

Methods: Here we examine data of cytokine, immune cell types, and disease associations captured from biomedical literature associated with COVID-19, Coronavirus in general, SARS, and H5N1 influenza, with the objective of identifying potentially useful relationships and areas for future research.

Results: Cytokine and cell-type associations captured from Medical Subject Heading (MeSH) terms linked to thousands of PubMed records, has identified differing patterns of associations between the four corpuses of publications (COVID-19, Coronavirus, SARS, or H5N1 influenza). Clustering of cytokine-disease co-occurrences in the context of Coronavirus has identified compelling clusters of co-morbidities and symptoms, some of which already known to be linked to COVID-19. Finally, network analysis identified sub-networks of cytokines and immune cell types associated with different manifestations, co-morbidities and symptoms of Coronavirus, SARS, and H5N1.
\end{abstract}

Conclusion: Systematic review of research in medicine is essential to facilitate evidence-based choices about health interventions. In a fast moving pandemic the approach taken here will identify trends and enable rapid comparison to the literature of related diseases.

Keywords: COVID-19, Coronavirus, SARS, H5N1 influenza, Cytokines, Haematopoietic cells

\section{Background}

The recent respiratory disease pandemic (COVID-19) [1], caused by the severe acute respiratory syndrome (SARS) Coronavirus 2 (SARS-CoV-2), has both high

\footnotetext{
*Correspondence: nophar.geifman@manchester.ac.uk

${ }^{1}$ Centre for Health Informatics, Division of Informatics, Imaging and Data Sciences, Faculty of Biology, Medicine and Health (FBMH), University of Manchester, Vaughan House, Portsmouth Street, Manchester M13 9GB, UK

Full list of author information is available at the end of the article
}

rates of infection as well as high mortality rates [2, 3]. In many inflammatory diseases, signaling proteins termed cytokines play a critical role in disease pathology. These proteins are secreted mainly from hematopoietic cells including lymphocytes and macrophages, and along with such cells, play a central role in many diseases as well as in health [4-7]. In the case of COVID-19, and other viral diseases, they can significantly affect the course and outcome for the patient. This may manifest itself as a cytokine release syndrome or cytokine storm, observed

c) The Author(s) 2020. This article is licensed under a Creative Commons Attribution 4.0 International License, which permits use, sharing, adaptation, distribution and reproduction in any medium or format, as long as you give appropriate credit to the original author(s) and the source, provide a link to the Creative Commons licence, and indicate if changes were made. The images or other third party material in this article are included in the article's Creative Commons licence, unless indicated otherwise in a credit line to the material. If material is not included in the article's Creative Commons licence and your intended use is not permitted by statutory regulation or exceeds the permitted use, you will need to obtain permission directly from the copyright holder. To view a copy of this licence, visit http://creativeco mmons.org/licenses/by/4.0/. The Creative Commons Public Domain Dedication waiver (http://creativecommons.org/publicdomain/ zero/1.0/) applies to the data made available in this article, unless otherwise stated in a credit line to the data. 
in many patients as highly elevated levels of these proteins during acute disease. While first linked to the 1918 influenza pandemic [8] the cytokine storm was extensively documented in the avian $\mathrm{H} 5 \mathrm{~N} 1$ influenza virus infection [9]. H5N1 influenza virus causes acute lung injury as also observed in COVID-19. Acute respiratory distress syndrome (ARDS) is the principal cause of respiratory failure associated with severe influenza as it is with COVID-19 and other members of the Coronavirus family. In severe influenza infections the extent of lung injury is due to dysregulated inflammatory responses. Hence, to compare and contrast immune-related trends in H5N1 influenza and Coronavirus infections could enable clinical research to more effectively consider treatment options.

SARS-CoV-2 is closely related to SARS-CoV, the virus responsible for the 2003 SARS pandemic. Since COVID19 is an emerging pandemic, there is currently less data available on the involvement of the immune system. However, the resource available for Coronaviruses more generally, SARS more specifically, as well as H5N1 influenza, makes it possible to gain insight into the pathology associated with SARS-CoV-2, in respect of pro-inflammatory events and lung damage.

One rich, readily available source of immune- and disease-related knowledge is the corpus of published scientific research. Within research publications, a copious amount of disease-related trends are captured; these can be freely extracted from PubMed records [10]. We have previously developed a framework for extracting disease-immune relationships from PubMed records by relying on the linking of such records to concepts from Medical Subject Headings (MeSH) [11]. MeSH is the National Library of Medicine's controlled vocabulary thesaurus, consisting of terms (naming and descriptors) within a hierarchical structure; they are used for indexing MEDLINE PubMed publications. MeSH descriptors associated with each MEDLINE citation are manually assigned and provide a straightforward, and useful, knowledge resource. Numerous works using concept co-occurrences in biomedical texts or in associated $\mathrm{MeSH}$ terms have shown the utility of $\mathrm{MeSH}$ in capturing biomedical knowledge [12-18]. Our own assessment of the ability of MeSH descriptors associated within the same PubMed record to represent a true (meaningful and feasible) relationship between the terms has shown that co-occurrence of $\mathrm{MeSH}$ descriptors linked to any given PubMed record are a good source for mining dependencies between different types of biomedical entities [11].

The work presented here examines immune-related molecular and cellular patterns in the context of COVID19. Coronaviruses, SARS, and in H5N1 influenza. By identifying links between these conditions and different immune system players, novel research can be better targeted at areas of greater impact.

\section{Methods}

\section{Extracting PubMed record-associated MeSH descriptors}

A complete description of the approach taken by this study can be found in [11]. Briefly, a list of cytokine MeSH descriptors (such as "interferon gamma," "transforming growth factor beta," and "chemokine CCL3") was manually compiled by a domain expert, who browsed MeSH's sub-trees and selected those descriptors that were deemed relevant to this work. Similarly, a list of immunerelated cell type $\mathrm{MeSH}$ descriptors (such as "lymphocytes," "Th1 cells," and "basophils") was also compiled. A comprehensive list of disease names and synonyms was extracted from the Human Disease Ontology.

For each PubMed record (see below) the list of associated MeSH descriptors was recorded. The lists of cytokine names, cell types, and diseases (described above) were then searched for exact matches within the $\mathrm{MeSH}$ descriptors associated with each PubMed record. Next, co-occurrences of cytokines, cell, or disease terms were searched for in each PubMed record. These associations and co-occurrences were then used for the analyses described below.

This approach to capturing associations between disease, cytokines and different cell types had been extensively evaluated, as described in [11]. Briefly, 100 randomly selected PubMed abstracts were manually evaluated by a domain expert who examined whether the co-occurrences of $\mathrm{MeSH}$ descriptors within the same PubMed record represent a true (meaningful and feasible) relationship between the terms. For each abstract, each pair of $\mathrm{MeSH}$ descriptors (disease-cell, diseasecytokine, or cell-cytokine) was evaluated to determine whether it represented a true relationship, an indirect relationship, or an incorrect/no relationship. Our evaluation found that over $70 \%$ of co-occurrences of different types of terms (disease, cell type, or cytokine) were found to represent true direct or indirect dependencies.

\section{PubMed data}

The PubMed database was searched on April 6th, 2020 for publications tagged with either the 'Severe Acute Respiratory Syndrome' MeSH term, the 'influenza a virus, h5n1 subtype' MeSH term, or with the 'Coronavirus' MeSH term (excluding those with the SARS Mesh term). These searches formed the SARS corpus with a total of 4493 records, the H5N1 corpus with a total of 5975 records, and the Coronavirus corpus with a total of 9810 records, respectively (Fig. 1). A further search using 
(See figure on next page.)

Fig. 1 Extraction of PubMed record-associated MeSH descriptors. The lower Venn diagrams represents the occurrences of the different types of MeSH descriptors (disease, cell, or cytokine), with overlapping areas representing the co-occurrences between the different term types. * PubMed was searched for publications linked to the 'Coronavirus' MeSH term but not to the SARS MeSH term. § Updated search of PubMed for Covid-19 related records conducted July 15 th 2020

the 'COVID-19' search term was conducted on July 15 th 2020 and resulted in a total of 6029 records.

\section{Clustering and correlation analysis}

In order to examine disease similarities based on cytokine or cell-type co-occurrences in the context of Coronavirus or SARS, we set out to cluster these patterns of cooccurrences in the literature. To do so, a quantitative cytokine-disease or cell-disease matrix was generated by obtaining, for each disease and cytokine/cell in the data, a count of the number of records mapped to that disease and that cytokine/cell.

Using these matrices as input, hierarchical clustering was performed using (1-correlation) as the distance measure.

\section{Network analysis}

Network analysis was carried out with the Cytoscape software. One network was created using the information extracted from the Coronavirus (but not SARS) corpus; a second network was created using data extract from the SARS corpus; and a third was created for H5N1. A fourth network was generated using data extracted from the COVID-19 corpus. Sub-networks were created by selecting nodes (primarily disease nodes) of interest and then selecting first-degree neighbors. Node and edge attributes such as, node type, node counts (of occurrences) and edge counts (of co-occurrences) were used for colouring, sizing, and layout of the networks.

\section{Results}

Using a previously published approach [11] associations between cytokines, immune cell types, and diseases were captured from PubMed records related to COVID-19, Coronavirus (in general), SARS and H5N1 influenza. Differing patterns of associations were identified in a corpus of over 9000 Coronavirus (excluding SARS) related publications and compared to those obtained in a corpus of over 4000 SARS related publications, and a corpus of nearly $6000 \mathrm{H} 5 \mathrm{~N} 1$ related publications.

\section{Different cytokines and cell types are associated with Coronavirus, SARS and H5N1 influenza}

In a comparison of the number of associations of cytokines and immune cells with publications between the four corpuses, some interesting differences were identified (Fig. 2 and Additional file 1). For example, Coronavirus is more highly associated with Interferon-gamma than SARS, H5N1 or COVID-19; while Interferon-beta is less associated with SARS-related publications than Coronavirus- or H5N1-related publications (Fig. 2a). On the other hand, SARS -related publications have a higher percentage of those linked to CXCL10 (interferon- $\gamma$ inducible protein 10) and Interleukin (IL)-8 (Fig. 2a). COVID-19 related publications show the highest association with IL-6; this may be the result of a focus of current research on cytokine storms in COVID-19 infections. Other differences between the four corpuses are found for different immune cells (Fig. 2b).

\section{Immune-related correlations between diseases associated with Coronavirus}

Clustering of cytokine-disease co-occurrences in the literature has potentially identified novel relationships between different diseases that are somehow related to Coronavirus.

For example, based on these patterns of cytokine associations, the common cold, asthma, and status asthmaticus (a severe form of repetitive asthma attacks) were clustered together (Fig. 3). A second cluster included hypertension, nephritis, chorioretinitis and uvetis, while a third interesting cluster included diabetes, hepatitis $\mathrm{C}$ and $\mathrm{B}$, panuveitis, pneumonia and pseudorabies. It should be noted however that there was a relatively small number of cytokine-disease co-occurrences in this corpus (when compared for example, to analysis of the entire PubMed database as in [11]).

\section{Immune interactions in comorbidities of Coronavirus, SARS} and H5N1 influenza

Network analysis of the interactions between the different entities captured from PubMed records has identified some interesting and differing sub-networks and connections. For example, in the network generated from COVID-19 related associations, interesting links were found between hypertension and IL-6, as well as around lymphopenia (Fig. 4). In Coronavirus-related associations (Fig. 5) different links between cytokines and immune cells and comorbidities or symptoms associated with Coronavirus can be seen. Where asthma and the common cold are directly linked to Interferon-gamma (IFNg), IL-8, IL-2 and IL-5, diarrhea is linked to IL-18. 


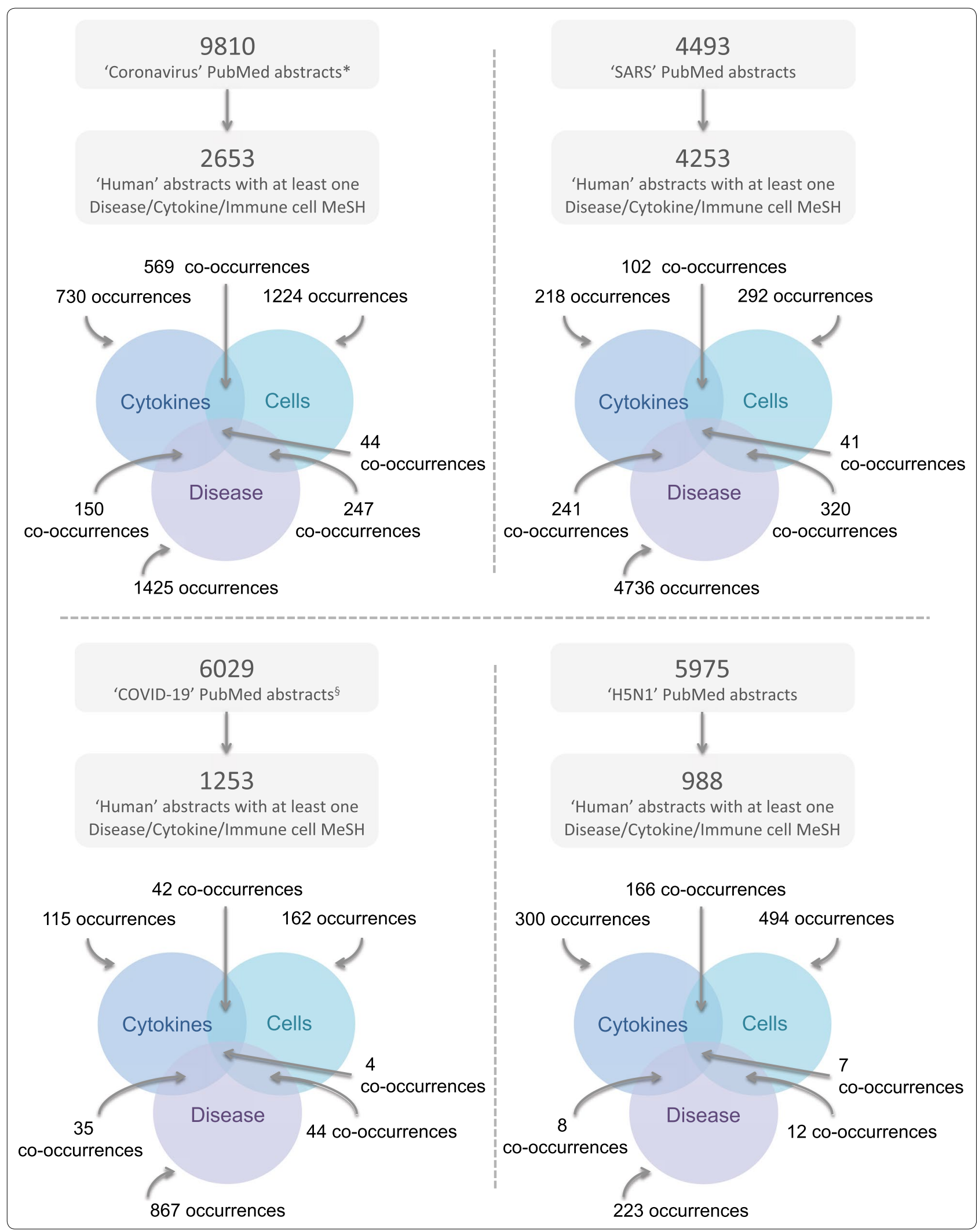




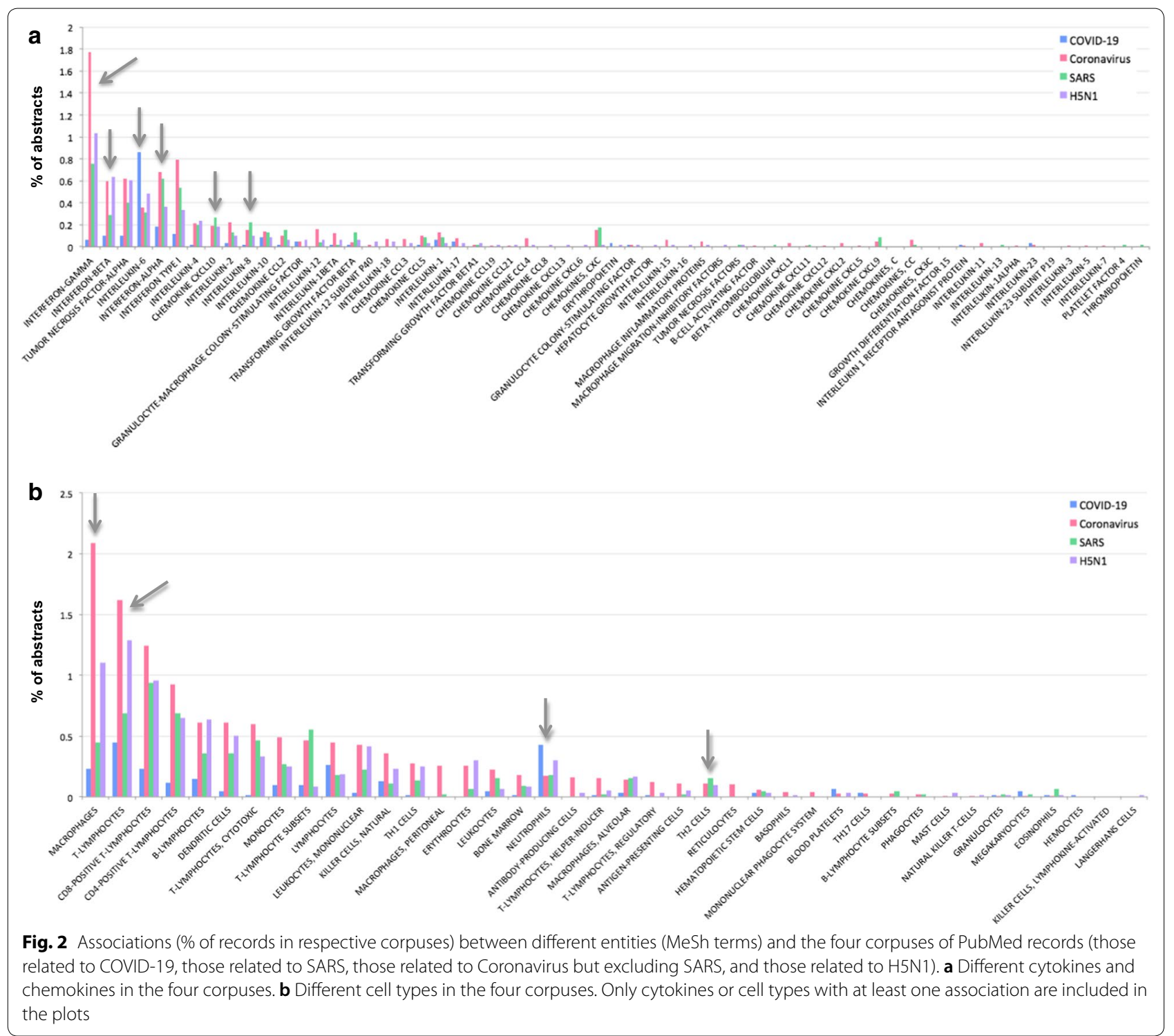

Differences in the types of cells included in each subnetwork can also be seen. Similarly, in the SARS-related network, we identify differing sub-networks each around a different SARS-related comorbidity (Fig. 6); as we did in the H5N1-related network (Fig. 7).

\section{Discussion}

In this work, we examined possible links of interest between cytokines, immune system cells and diseases in the context of Coronavirus and related infections. By mining information captured within biomedical publications, in the form of MeSH descriptors, and their cooccurrences, we identified associations that may warrant further investigations.
As the literature on SARS-COV-2 develops our approach can be reapplied to identify the strongest, direct links.

In examining the associations between cell types and cytokines, in PubMed records linked to COVID-19, Coronavirus (but not SARS), and those linked to SARS, we found some differences that may point to aetiological differences between SARS-CoV and Coronaviruses more generally. Coronavirus-related publications had a significantly higher percentage of associations with Interferon-gamma, Interferon-beta, T-lymphocytes, and macrophages (Fig. 2). On the other hand, SARS-related publications had higher associations with $\mathrm{C}-\mathrm{X}-\mathrm{C}$ motif chemokine 10 (CXCL10), IL8, and TH2 cells; however, these were only marginally higher than the number of 


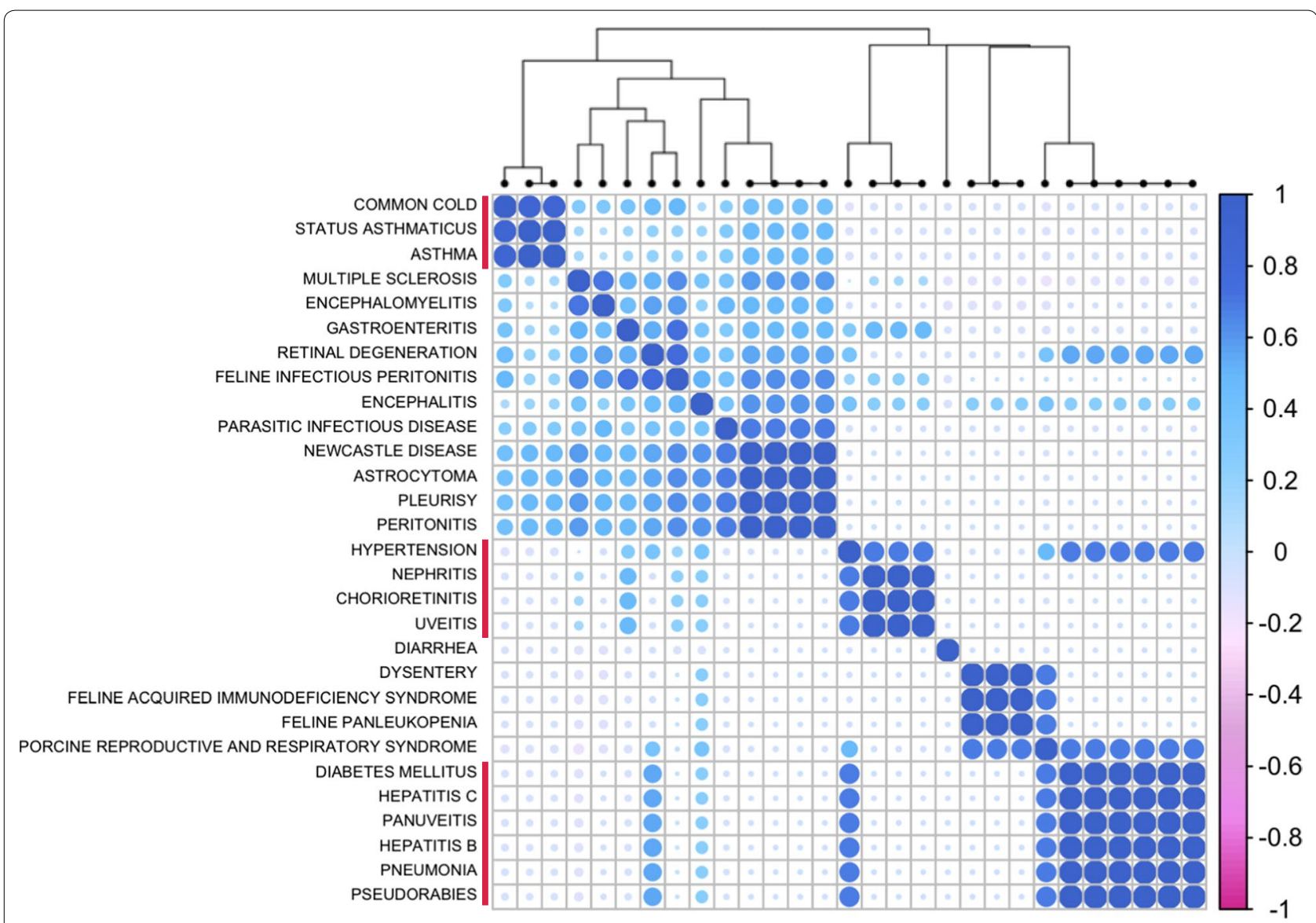

Fig. 3 Hierarchical clustering of diseases linked to Coronavirus-related publications, based on their patterns of associations with different cytokines

associations found in the Coronavirus-related corpus. COVID-19 related publications were highly associated with IL-6, reflective of the empirical finding of IL-6 elevation in this disease that in turn relates to cytokine release syndrome mooted as a mechanism underlying more severe cases of the disease [19-22]. Interestingly, COVID-19 publications also showed the highest association with the specific hematopoietic cell type, neutrophils (activated by and responsive to numerous cytokines). When comparing these cytokine and cell occurrences to those in H5N1-related publications, some patterns are similar to those observed in the Coronavirus-related publications; specifically, similar rates of occurrences were observed for IFNb, TNFa, IL-18, and GranulocyteMacrophage Colony Stimulating Factor (GMCSF), in all cases these were higher than in the SARS-related publications. On the other hand H5N1-related publications had similar rates of association for monocytes, CD8-positive, and CD4-positive T-lymphocytes to those in SARS, in all cases, lower than those in Coronavirus-related publications but higher than in COVID-19 related publications.
While we cannot rule out that these differences between the four corpuses are partially due to noise, or random variance, it is likely that differences in research focus reflect an underlying cause, and this may reflect biological or clinical differences. The clearest picture of the key features of COVID-19 disease becomes apparent by a consideration of all relevant publications and comparison to related diseases. Here we have shown this is applicable immediately.

Hierarchical clustering of diseases linked to Coronavirus-related publications has also identified some interesting groupings of diseases and symptoms associated with Coronavirus infections. For example, the common cold, asthma, and status asthmaticus, are all highly correlated based on their pattern of associations with cytokines within the Coronavirus-related corpus (Fig. 3). In a second example, hypertension, nephritis, chorioretinitis and uveitis are all clustered together; and in a third example, diabetes, hepatitis $\mathrm{C}$ and $\mathrm{B}$, panuveitis, pseudorabies all cluster with pneumonia. These clusters may suggest 


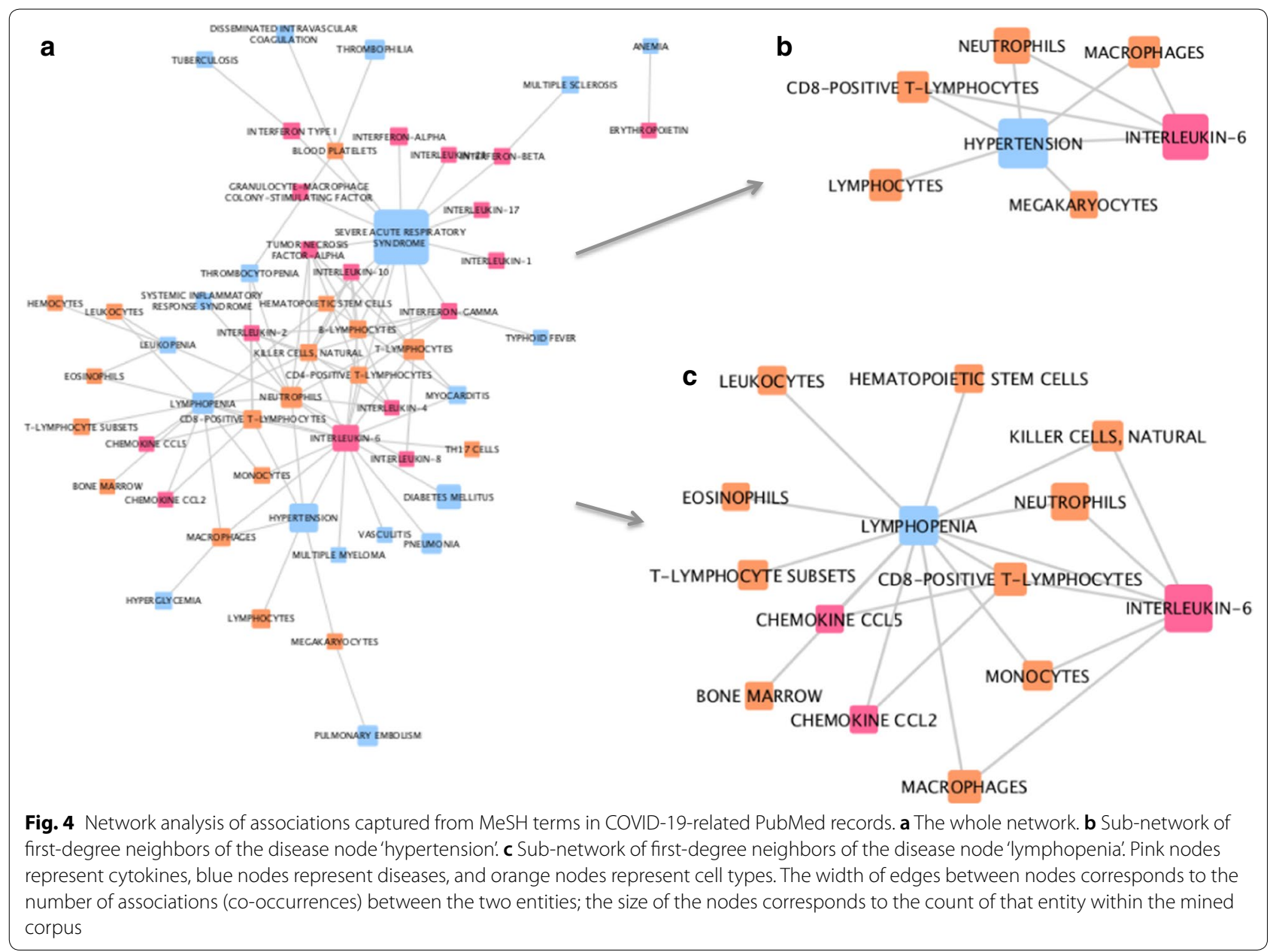

similar underlying immunological mechanisms in the context of coronaviruses.

In our third analysis, networks illustrating the connections between cytokines, immune cell types and different diseases, captured from the four publication corpuses, were generated. Sub-networks, each stemming from a selected disease, illustrate some differences between COVID-19 -, Coronavirus-, SARS-, and H5N1-related comorbidities and complications. For example, in Coronavirus-related publications, diarrhea, one of the reported symptoms of COVID-19, is associated with IL-18, monocytes, T-lymphocytes, dendritic cells and erythrocytes. On the other hand, asthma, a risk factor for adverse outcomes in COVID-19, is linked to IL-8, IL-5, IL-2, IFNg, eosinophils, and neutrophils. In the COVID-19 related network, though based on a limited number of associations, hypertension is highlighted (Fig. 4b); this is now known to be a comorbidity associated with increased risk of infection and worse outcomes of COVID-19 [23, 24], validating our approach.
This analysis has highlighted some potential targets for therapy. For example, IL-5 is a key growth factor for and activator of eosinophils (both picked up by our research). Eosinophils can be recruited to the lungs, where they have a poorly understood role in health and disease [25]. It has also been reported eosinophil count is a potential marker for COVID-19 patient improvement [26, 27]. The role of eosinophils and IL- 5 in this disease certainly requires further investigation. Treatments for asthma that modulate eosinophil action are available; Reslizumab and Mepolizumab are anti-IL-5 antibody therapies that may have potential in treatment of viral respiratory disease, if eosinophils are activated [28, 29]. It is speculative to comment on the role of eosinophils in COVID-19 disease; however in overall consideration of a fast developing literature in a pandemic many possible clinical trial options could be considered. As the literature evolves we suggest the methodologies described here can play a progressive part in rational development of strategies for optimal treatment in an informed environment. 


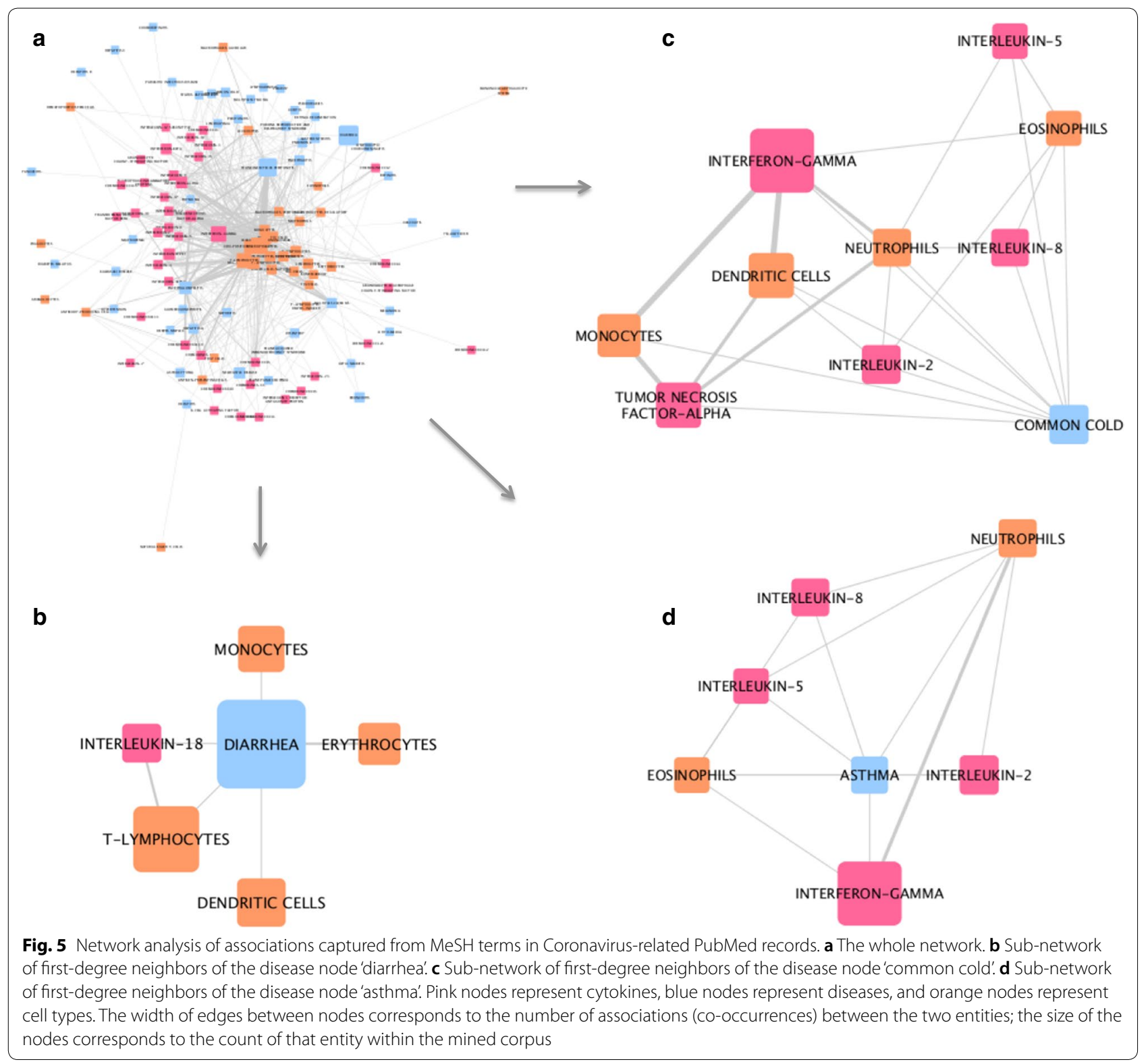

Our approach to capturing immune-related associations from $\mathrm{MeSH}$ descriptors is not without limitations. We first assume that co-occurrences of $\mathrm{MeSH}$ descriptors within a PubMed record represent a true relationship or dependency; further, some of the associations and cooccurrences were low (i.e. present in only few records). However, our previous investigation into the extent to which $\mathrm{MeSH}$ term co-occurrence captures real association has found that at least $70 \%$ of co-occurrences of different types of entities (disease, cell type, or cytokine) represent true direct or indirect dependencies, but it is likely to be higher than that [11]. Additionally, patterns of $\mathrm{MeSH}$ co-occurrence have shown to capture known medical associations, as well as identify potentially novel ones, thus providing further confidence in the approach. A second limitation is a lack of directionality and type for the associations captured by approach; nevertheless, we show that these mere co-occurrences may still hold valuable information. Finally, at the time of writing, too few publications directly related to COVID-19 that reported immune involvement and mechanisms are available in PubMed. While many COVID-19 publications are 


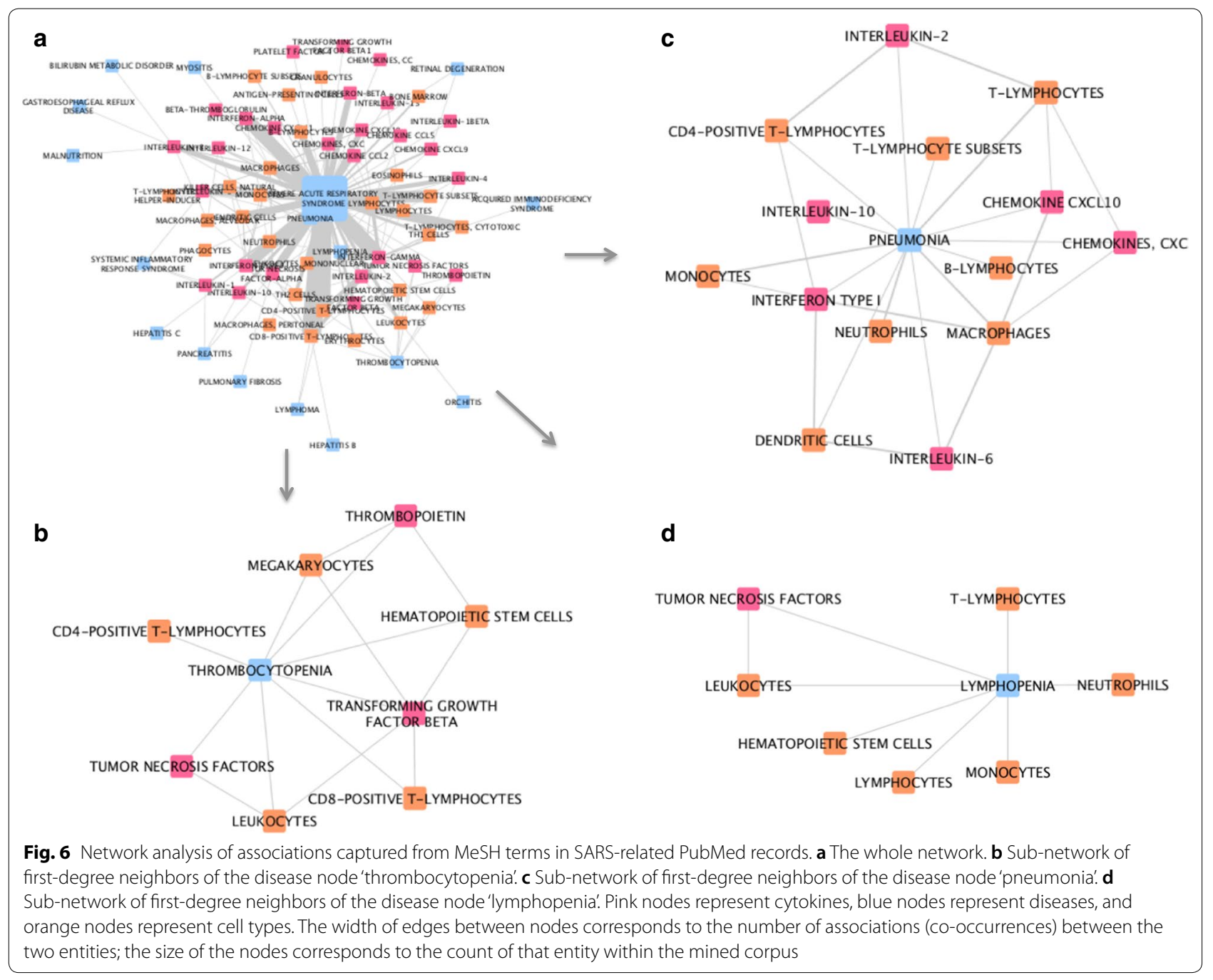

available in preprint repositories these could not be mined by our approach which relies on the curated association with $\mathrm{MeSH}$. The consensus view on scientific method leads us to argue our method is better applied to peer reviewed publications. While our approach did find some associations in our mined COVID-19 corpus, our data mining in Coronavirus- and other related lung-damaging diseases as a proxy for COVID-19 produced valid comparators in terms of drug targeting and an overview of molecular cellular pathology. We recently performed an analysis of protein biomarkers in COVID19 [30]; this labour-intensive approach produced similar data to the methodology we describe here. Thus, we have 


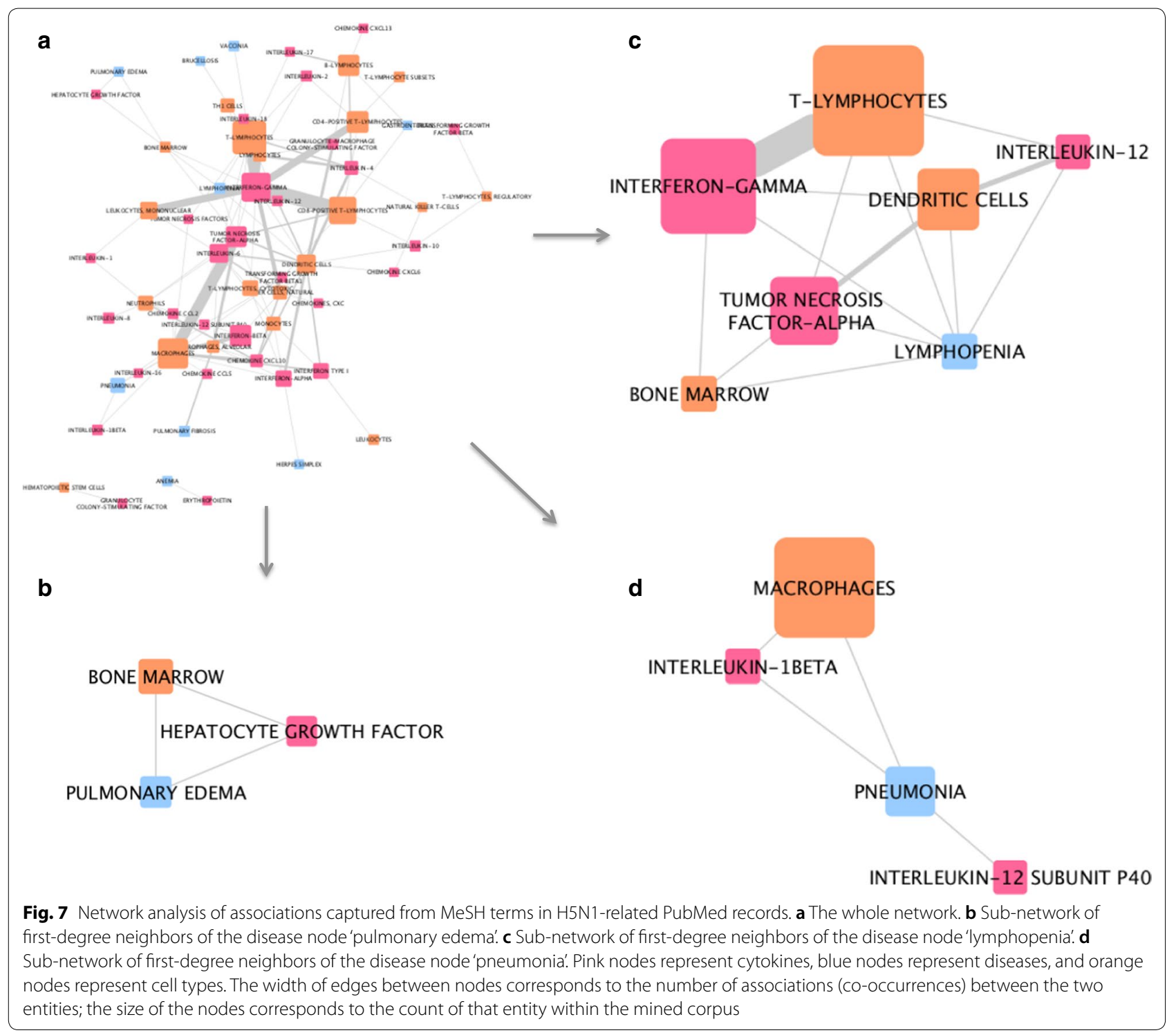

created a paradigm for such research which is easy to use and apply, and demonstrated its utility.

\section{Conclusion}

Herein, we have identified possible links between immune-related patterns, related co-morbidities, complications and symptoms in the context of Coronavirus and related infections. These associations may have direct implications for COVID-19 and can help focus on potentially useful avenues of future research to understanding of the immune mechanisms underlying COVID-19 and related complications.

\section{Supplementary information}

Supplementary information accompanies this paper at https://doi. org/10.1186/s12967-020-02472-z.

Additional file 1: a) Occurrences of cytokine MeSh terms in the four corpuses. b) Occurrences of cell-type MeSh terms in the four corpuses. c) Occurrences of disease MeSh terms in the four corpuses (only diseases with $>1$ occurrences are shown). 


\begin{abstract}
Abbreviations
SARS: Severe acute respiratory syndrome; MeSH: Medical Subject Heading: ARDS: Acute respiratory distress syndrome; IL: Interleukin; IFN: Interferon; GMCSF: Granulocyte-Macrophage Colony Stimulating Factor; CXCL10: C-X-C motif chemokine 10; TNF: Tumor necrosis factor.
\end{abstract}

\section{Acknowledgements}

This work was supported by the NIHR Manchester Biomedical research Centre.

\section{Authors' contributions}

NG conceived the idea for the study and designed it together with ADW. NG carried out the analysis presented in this paper. NG and ADW both wrote the manuscript. Both authors read and approved the final manuscript.

\section{Funding}

Not applicable.

\section{Availability of data and materials}

All data are available in Additional file 1.

Ethics approval and consent to participate

Not applicable.

\section{Consent for publication}

Not applicable.

\section{Competing interests}

The authors declare that they have no competing interests.

\section{Author details}

${ }^{1}$ Centre for Health Informatics, Division of Informatics, Imaging and Data Sciences, Faculty of Biology, Medicine and Health (FBMH), University of Manchester, Vaughan House, Portsmouth Street, Manchester M13 9GB, UK. ${ }^{2}$ Stoller Biomarker Discovery Centre, Division of Cancer Sciences, FBMH, University of Manchester, Manchester, UK. ${ }^{3}$ Manchester National Institute for Health Biomedical Research Centre, Manchester, UK.

Received: 11 May 2020 Accepted: 28 July 2020

Published online: 03 August 2020

\section{References}

1. Huang C, Wang Y, Li X, Ren L, Zhao J, Hu Y, Zhang L, Fan G, Xu J, Gu X, et al. Clinical features of patients infected with 2019 novel coronavirus in Wuhan, China. Lancet. 2020:395(10223):497-506.

2. Li Q, Guan X, Wu P, Wang X, Zhou L, Tong Y, Ren R, Leung KSM, Lau EHY, Wong JY, et al. Early transmission dynamics in wuhan, china, of novel Coronavirus-infected pneumonia. N Engl J Med. 2020;382(13):1199-207.

3. Verity R, Okell LC, Dorigatti I, Winskill P, Whittaker C, Imai N, Cuomo-Dannenburg G, Thompson H, Walker PGT, Fu H, et al. Estimates of the severity of coronavirus disease 2019: a model-based analysis. Lancet Infect Dis. 2020;20:669-77

4. Cromheecke JL, Nguyen KT, Huston DP. Emerging role of human basophil biology in health and disease. Curr Allergy Asthma Rep. 2014;14(1):408.

5. Hamze M, Desmetz C, Guglielmi P. B cell-derived cytokines in disease. Eur Cytokine Netw. 2013;24(1):20-6.

6. Melo RC, Liu L, Xenakis JJ, Spencer LA. Eosinophil-derived cytokines in health and disease: unraveling novel mechanisms of selective secretion. Allergy. 2013;68(3):274-84.

7. Wu L, Van Kaer L. Natural killer T cells in health and disease. Front Biosci (Schol Ed). 2011;3:236-51.

8. Short KR, Kedzierska K, van de Sandt CE. Back to the future: lessons learned from the 1918 influenza pandemic. Front Cell Infect Microbiol. 2018;8:343.

9. Cheung CY, Poon LL, Lau AS, LukW, Lau YL, Shortridge KF, Gordon S, Guan $Y$, Peiris JS. Induction of proinflammatory cytokines in human macrophages by influenza $\mathrm{A}$ (H5N1) viruses: a mechanism for the unusual severity of human disease? Lancet. 2002;360(9348):1831-7.

10. National Library of Medicine (NLM), National Center for Biotechnology Information (NCBI). PubMed [Internet]. http://www.ncbi.nlm.nih.gov/ pubmed/. Accessed 6 April 2020.
11. Geifman N, Bhattacharya S, Butte AJ. Immune modulators in disease: integrating knowledge from the biomedical literature and gene expression. J Am Med Inform Assoc. 2016;23(3):617-26.

12. Avillach $P$, Dufour JC, Diallo G, Salvo F, Joubert M, Thiessard F, Mougin F, Trifiro G, Fourrier-Reglat A, Pariente A, et al. Design and validation of an automated method to detect known adverse drug reactions in MEDLINE: a contribution from the EU-ADR project. J Am Med Inform Assoc. 2013;20(3):446-52.

13. Cimino JJ, Barnett GO. Automatic knowledge acquisition from MEDLINE. Methods Inf Med. 1993;32(2):120-30.

14. Doerks T, van Noort V, Minguez P, Bork P. Annotation of the M. tuberculosis hypothetical orfeome: adding functional information to more than half of the uncharacterized proteins. PLoS One. 2012;7(4):e34302.

15. Kastrin A, Rindflesch TC, Hristovski D. Large-scale structure of a network of co-occurring MeSH terms: statistical analysis of macroscopic properties. PLoS ONE. 2014;9(7):e102188.

16. Kavuluru R, Lu Y. Leveraging output term co-occurrence frequencies and latent associations in predicting medical subject headings. Data Knowl Eng. 2014;94(B):189-201.

17. Srinivasan $P$, Rindflesch T. Exploring text mining from MEDLINE. Proc AMIA Symp 2002; 722-726.

18. Stapley BJ, Benoit G. Biobibliometrics: information retrieval and visualization from co-occurrences of gene names in Medline abstracts. Pac Symp Biocomput. 2000. https://doi.org/10.1142/9789814447331_0050.

19. Zhang C, Wu Z, Li JW, Zhao H, Wang GQ. Cytokine release syndrome in severe COVID-19: interleukin-6 receptor antagonist tocilizumab may be the key to reduce mortality. Int J Antimicrob Agents. 2020;55(5):105954.

20. McGonagle D, Sharif K, O'Regan A, Bridgewood C. The role of cytokines including interleukin-6 in COVID-19 induced pneumonia and macrophage activation syndrome-like disease. Autoimmun Rev. 2020;19(6):102537.

21. Ulhaq ZS, Soraya GV. Interleukin-6 as a potential biomarker of COVID-19 progression. Med Mal Infect. 2020;50(4):382-3.

22. Grifoni E, Valoriani A, Cei F, Lamanna R, Gelli AMG, Ciambotti B, Vannucchi V, Moroni F, Pelagatti L, Tarquini R, et al. Interleukin-6 as prognosticator in patients with COVID-19. J Infect. 2020. https://doi.org/10.1016/j. jinf.2020.06.008.

23. Fang L, Karakiulakis G, Roth M. Are patients with hypertension and diabetes mellitus at increased risk for COVID-19 infection? Lancet Respir Med. 2020;8(4):e21.

24. Schiffrin EL, Flack JM, Ito S, Muntner P, Webb RC. Hypertension and COVID-19. Am J Hypertens. 2020:33(5):373-4.

25. Jacobsen EA, Helmers RA, Lee JJ, Lee NA. The expanding role(s) of eosinophils in health and disease. Blood. 2012;120(19):3882-90.

26. Cao X. COVID-19: immunopathology and its implications for therapy. Nat Rev Immunol. 2020;20(5):269-70.

27. Liu F, Xu A, Zhang Y, Xuan W, Yan T, Pan K, Yu W, Zhang J. Patients of COVID-19 may benefit from sustained lopinavir-combined regimen and the increase of eosinophil may predict the outcome of COVID-19 progression. Int J Infect Dis. 2020;95:183.

28. Castro M, Mathur S, Hargreave F, Boulet LP, Xie F, Young J, Wilkins HJ, Henkel T, Nair P. Res-5- Study G: reslizumab for poorly controlled, eosinophilic asthma: a randomized, placebo-controlled study. Am J Respir Crit Care Med. 2011;184(10):1125-32

29. Henriksen DP, Bodtger U, Sidenius K, Maltbaek N, Pedersen L, Madsen $H$, Andersson EA, Norgaard O, Madsen LK, Chawes BL. Efficacy, adverse events, and inter-drug comparison of mepolizumab and reslizumab anti-IL-5 treatments of severe asthma-a systematic review and metaanalysis. Eur Clin Respir J. 2018;5(1):1536097.

30. Whetton AD, Preston GW, Abubeker S, Geifman N. Proteomics and informatics for understanding phases and identifying biomarkers in COVID-19 disease. J Proteome Res. 2020. https://doi.org/10.1021/acs.jprot eome.0c00326.

\section{Publisher's Note}

Springer Nature remains neutral with regard to jurisdictional claims in published maps and institutional affiliations. 